\title{
Synthetic lon Channels with Rigid-Rod ח-Stack Architecture that Open in Response to Charge-Transfer Complex Formation
}

\author{
Pinaki Talukdar, Guillaume Bollot, Jiri Mareda, Naomi Sakai and Stefan Matile \\ Department of Organic Chemistry, University of Geneva, Geneva, Switzerland \\ stefan.matile@chiorg.unige.ch
}

\section{Supporting Information}

General. Reagents for synthesis were purchased from Fluka, amino acid derivatives from Novabiochem and Bachem, egg yolk phosphatidylcholine (EYPC) from Avanti polar lipids, HATU from Applied Biosystems, HPTS, ANTS and DPX from Molecular Probes, gramicidin, buffers, and salts from Sigma or Fluka-Aldrich. All reactions were performed under argon atmosphere. Unless stated otherwise, column chromatography was carried out on silica gel 60 (Fluka, 40-63 $\mu \mathrm{m}$ ). Reverse phase column chromatography was performed using ODS (Fluka Silicagel 100 C18-Reverse Phase). Analytical (TLC) and preparative thin layer chromatography (PTLC) were performed in silica gel 60 (Fluka, $0.2 \mathrm{~mm}$ ) and silica gel GF (Analtech, $1000 \mu \mathrm{m}$ ), respectively. Sephadex LH-20 was from Amersham Biosciences. HPLC was performed using either Jasco HPLC system (PU-980, UV-970, FP-920) or an Agilent 1100 series apparatus with a photo diode array detector. $[\alpha]_{D}^{20}$ values were recorded on a Jasco P-1030 Polarimeter, melting points (m.p.) on a heating table from Reichert (Austria), elemental analysis on a Vario EL instrument from Elementar. IR spectra were recorded on a Perkin Elmer Spectrum One FT-IR spectrometer (ATR, Golden Gate, unless stated) and are reported as wavenumbers $v$ in $\mathrm{cm}^{-1}$ with band intensities indicated as $\mathrm{s}$ (strong), m (medium), w (weak). ESI-MS and APCI-MS were performed on a Finnigan MAT SSQ 7000 instrument, EI-MS on a VG Analytical 7070E Mass Spectrometer. ${ }^{1} \mathrm{H}$ and ${ }^{13} \mathrm{C}$ spectra were recorded (as indicated) either on a Bruker $300 \mathrm{MHz}, 400 \mathrm{MHz}$ or $500 \mathrm{MHz}$ spectrometer and are reported as chemical shifts $(\delta)$ in ppm relative to TMS $(\delta=0)$. Spin multiplicities are reported as a singlet (s), doublet (d), triplet (t), quartet (q) and quintet (quint) with coupling constants $(J)$ given in $\mathrm{Hz}$, or multiplet $(\mathrm{m})$. Broad peaks are marked as br. ${ }^{1} \mathrm{H}$ and ${ }^{13} \mathrm{C}$ resonances were assigned with the aid of additional information from $2 \mathrm{D}$ NMR spectra (H,H-COSY, DEPT 135, HSQC and HMBC). UV-Vis spectra were measured on a Varian Cary 1 Bio spectrophotometer equipped with a stirrer and a temperature controller (25 ${ }^{\circ} \mathrm{C}$ ) and are reported as maximal absorption wavelength $\lambda$ in $\mathrm{nm}$ (extinction coefficient $\varepsilon$ in $\mathrm{mM}^{-1} \mathrm{~cm}^{-1}$ ). CD spectra were obtained using a Jasco J-715 spectropolarimeter with a thermostated cell holder $\left(25{ }^{\circ} \mathrm{C}\right.$ or as indicated) and are reported as maximal absorption wavelength $\lambda$ in $\mathrm{nm}\left(\Delta \varepsilon\right.$ in $\left.\mathrm{M}^{-1} \mathrm{~cm}^{-1}\right)$. Fluorescence measurements were preformed either on a FluoroMax-2 or a FluoroMax-3, Jobin Yvon-Spex, both equipped with an injector port, a stirrer and a temperature controller $\left(25^{\circ} \mathrm{C}\right)$. The Mini-Extruder with a polycarbonate membrane, pore size $100 \mathrm{~nm}$, used for LUV preparation was from Avanti polar lipids.

Abbreviations. ANTS: 8-Aminonaphthalene-1,3,6-trisulfonic acid, disodium salt; Boc: tert-Butoxycarbonyl; DAN: Dialkoxynaphthalene; DMF: $N, N$-Dimethylformamide; DPX: 
p-Xylene-bis-pyridinium bromide; EYPC LUVs: Egg yolk phosphatidylcholine large unilamellar vesicles; Gla: $-\mathrm{OCH}_{2} \mathrm{CO}-(\mathrm{H}-\mathrm{Gla}-\mathrm{OH}$ : glycolic acid); HATU: $N$ [(Dimethylamino)-1H-1,2,3-triazolo[4,5-b]pyridin-1-ylmethylene]- $N$-methylmethanaminium hexafluorophosphate $\mathrm{N}$-oxide; HEPES: 4-(2-Hydroxyethyl)-1-piperazine-ethanesulfonic acid; HPTS: 8-Hydroxy-1,3,6-pyrenetrisulfonate; Lys: L-Lysine; NDI: Naphthalenediimide; RPHPLC: Reverse phase high performance liquid chromatography; rt: Room temperature; TEA: Triethylamine; TES: 2[(2-Hydroxy-1,1-bis[hydroxymethyl]ethyl)aminoethanesulfonic acid; TFA: Trifluoroacetic acid; Z: (Benzyloxy)carbonyl.

\section{Synthesis and Characterization of $1^{3}, 2^{3}, 3^{2}, 4^{3}, 5^{2}, 6^{3}, 7^{2}, 8^{3}$-Octakis(NDI-Lys-NH $)$-p- Octiphenyl 1.}

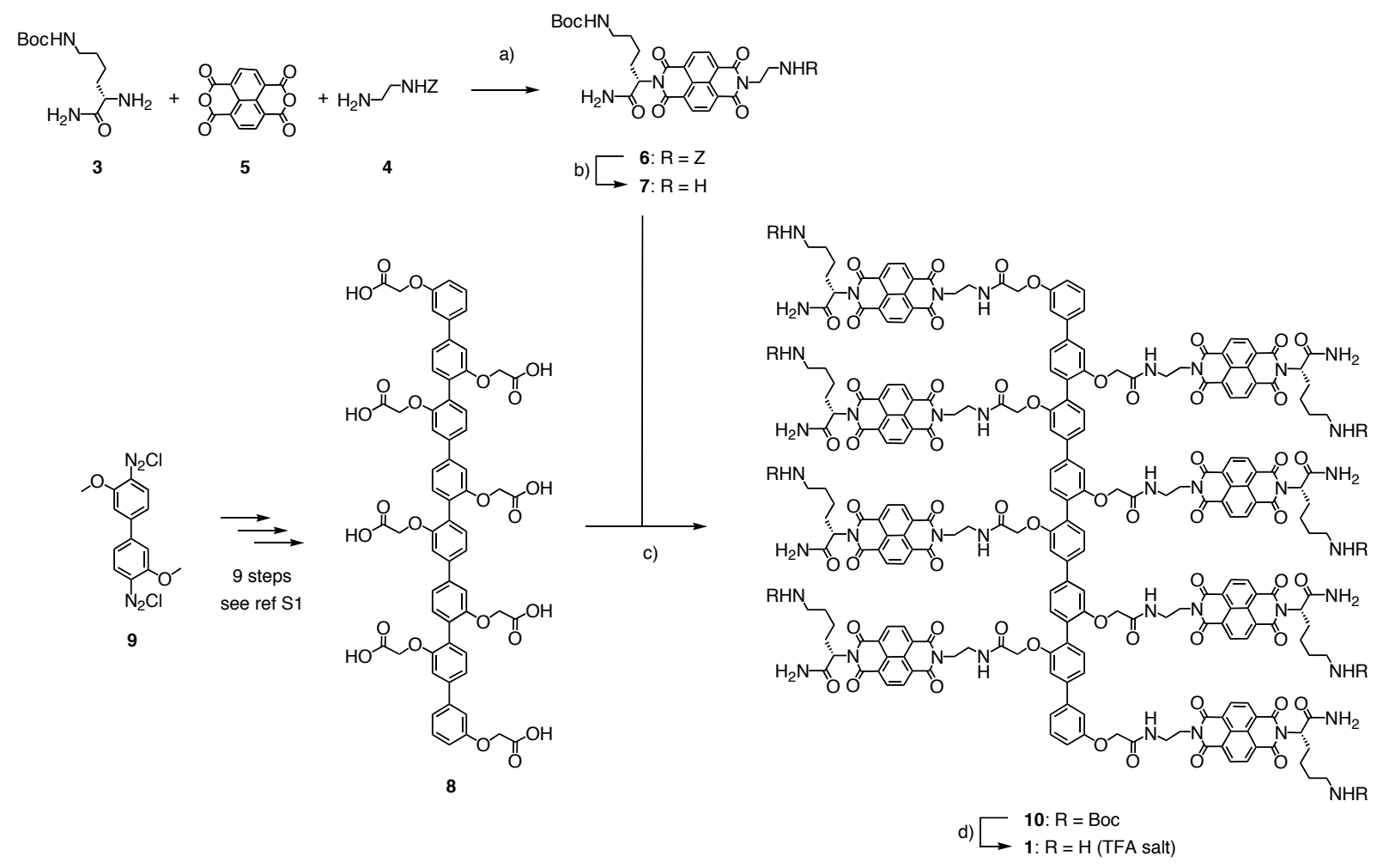

Scheme S1. a) TEA, DMF, 48 h, $40{ }^{\circ} \mathrm{C}, 50 \%$; b) $\mathrm{H}_{2}, \mathrm{Pd}(\mathrm{OH})_{2} / \mathrm{C}, \mathrm{MeOH}, 6$ h, rt, $91 \%$; c) HATU, DMF, 2,6-di-tert-butyl pyridine, $24 \mathrm{~h}$, rt, 59\%; d) TFA/ $\mathrm{CH}_{2} \mathrm{Cl}_{2} 1: 1,73 \%$.

Z-NDI-Lys(Boc)-NH $\mathbf{N H}_{2}$. To a solution of $3(504 \mathrm{mg}, 1.79 \mathrm{mmol})$ in dry DMF $(25 \mathrm{ml}), \mathbf{4}$ (413 mg, $1.79 \mathrm{mmol}), 5$ (400 $\mathrm{mg}, 1.49 \mathrm{mmol})$ and TEA ( $1 \mathrm{ml}, 4.78 \mathrm{mmol})$ were added. The reaction mixture was stirred at $40{ }^{\circ} \mathrm{C}$ for $48 \mathrm{~h}$. After evaporation of the solvent under reduced pressure, the crude material was purified by column chromatography $\left(\mathrm{CH}_{2} \mathrm{Cl}_{2} / \mathrm{MeOH} 25: 1, R_{f}\right.$ $=0.3)$ to give $6(495 \mathrm{mg}, 50 \%)$ as a brown solid. $[\alpha]_{\mathrm{D}}^{20}=-19.4\left(\mathrm{c}=1.0\right.$ in $\mathrm{CH}_{2} \mathrm{Cl}_{2} / \mathrm{MeOH}$ 1:1); m.p.: 113.5-114.0 ${ }^{\circ} \mathrm{C}$; IR: v 3346 (m), 3183 (m), 2934 (m), 1704 (s), 1659 (s), 1581 (s), 1516 (m), 1332 (s), 1245 (s), 1166 (s), 768 (s), 697 (s); ${ }^{1} \mathrm{H}$ NMR (500 MHz, CD $\left.{ }_{3} \mathrm{OD}\right): \delta 8.71$ $\left(\mathrm{d},{ }^{3} J(\mathrm{H}, \mathrm{H})=7.6 \mathrm{~Hz}, 2 \mathrm{H}\right), 8.66\left(\mathrm{~d},{ }^{3} J(\mathrm{H}, \mathrm{H})=7.6 \mathrm{~Hz}, 2 \mathrm{H}\right), 7.25-7.17(\mathrm{~m}, 4 \mathrm{H}), 7.10-7.01(\mathrm{~m}$, $1 \mathrm{H}), 5.63\left(\mathrm{dd},{ }^{3} J(\mathrm{H}, \mathrm{H})=9.5 \mathrm{~Hz},{ }^{3} J(\mathrm{H}, \mathrm{H})=5.5 \mathrm{~Hz}, 1 \mathrm{H}\right), 4.90(\mathrm{~s}, 2 \mathrm{H}), 4.32\left(\mathrm{t},{ }^{3} J(\mathrm{H}, \mathrm{H})=5.6\right.$ $\mathrm{Hz}, 2 \mathrm{H}), 3.51\left(\mathrm{t},{ }^{3} J(\mathrm{H}, \mathrm{H})=5.6 \mathrm{~Hz}, 2 \mathrm{H}\right), 2.96\left(\mathrm{t},{ }^{3} J(\mathrm{H}, \mathrm{H})=6.7 \mathrm{~Hz}, 2 \mathrm{H}\right), 2.35-2.23(\mathrm{~m}, 2 \mathrm{H})$, 1.59-1.50 (m, 1H), 1.48-1.39 (m, 1H), 1.41-1.27 (m, 2H), $1.30(\mathrm{~s}, 9 \mathrm{H}) ;{ }^{13} \mathrm{C}$ NMR $(125 \mathrm{MHz}$, $\left.\mathrm{CD}_{3} \mathrm{OD}\right): \delta 175.5(\mathrm{~s}), 165.4(\mathrm{~s}, \mathrm{x} 2), 165.3$ (s, x2), 160.0 (s), $159.4(\mathrm{~s}) 139.1$ (s), 132.8 (d, x2), 
$132.6(\mathrm{~d}, \mathrm{x} 2), 130.2(\mathrm{~d}, \mathrm{x} 2), 129.7$ (d), 129.5 (d, x2), 129.1 (s, x2), $129.0(\mathrm{~s}, \mathrm{x} 2), 128.8$ (s, x2), $80.6(\mathrm{~s}), 68.1(\mathrm{t}), 56.7(\mathrm{~d}), 42.3(\mathrm{t}), 41.8(\mathrm{t}), 40.7(\mathrm{t}), 31.7(\mathrm{t}), 30.0(\mathrm{t}), 29.6(\mathrm{q}, \mathrm{x} 3), 24.8(\mathrm{t})$; MS (ESI, $\left.\mathrm{CH}_{2} \mathrm{Cl}_{2} / \mathrm{MeOH} 9: 1\right): \mathrm{m} / \mathrm{z}(\%) 1365\left(49,[2 \mathrm{M}+\mathrm{Na}]^{+}\right), 694\left(100,[\mathrm{M}+\mathrm{Na}]^{+}\right)$.

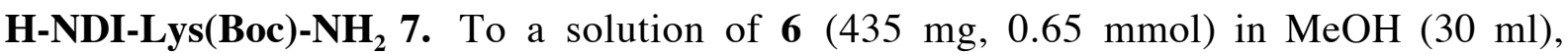
$\mathrm{Pd}(\mathrm{OH})_{2} / \mathrm{C}(5 \mathrm{mg})$ was added. The suspension was degassed and set under $\mathrm{H}_{2}$-atmosphere for $6 \mathrm{~h}$ at $\mathrm{rt}$, then filtered (celite) and concentrated in vacuo. Column chromatography $\left(\mathrm{CH}_{2} \mathrm{Cl}_{2} / \mathrm{MeOH} 15: 1\right.$, then $\mathrm{CH}_{2} \mathrm{Cl}_{2} / \mathrm{MeOH} / \mathrm{Et}_{3} \mathrm{~N}$ 100:10:1; $\left.\mathrm{CH}_{2} \mathrm{Cl}_{2} / \mathrm{MeOH} 4: 1, R_{f}=0.4\right)$ of the crude product yielded pure $7(318 \mathrm{mg}, 91 \%)$ as a brown solid. $[\alpha]_{D}^{20}=-24.4(\mathrm{c}=1.0 \mathrm{in}$ $\mathrm{MeOH}$ ); m.p.: 122.5- $123.0{ }^{\circ} \mathrm{C}$; IR: $v 3318$ (m), 3168 (m), 2935 (m), 1704 (s), 1660 (s), 1581 (s), 1516 (m), 1453 (s), 1332 (s), 1247 (s), 1164 (s), 768 (s); ${ }^{1} \mathrm{H}$ NMR (500 MHz, CD 3 OD): $\delta$ $8.70\left(\mathrm{~d},{ }^{3} J(\mathrm{H}, \mathrm{H})=7.6 \mathrm{~Hz}, 2 \mathrm{H}\right), 8.66\left(\mathrm{~d},{ }^{3} J=7.6 \mathrm{~Hz}, 2 \mathrm{H}\right), 5.61\left(\mathrm{dd},{ }^{3} J(\mathrm{H}, \mathrm{H})=9.8 \mathrm{~Hz},{ }^{3} J\right.$ $(\mathrm{H}, \mathrm{H})=5.2 \mathrm{~Hz}, 1 \mathrm{H}), 4.24\left(\mathrm{t},{ }^{3} J(\mathrm{H}, \mathrm{H})=6.5 \mathrm{~Hz}, 2 \mathrm{H}\right), 3.00\left(\mathrm{t},{ }^{3} J=6.5 \mathrm{~Hz}, 2 \mathrm{H}\right), 2.97\left(\mathrm{t},{ }^{3} J\right.$ $(\mathrm{H}, \mathrm{H})=7.0 \mathrm{~Hz}), 2.34-2.21(\mathrm{~m}, 2 \mathrm{H}), 1.59-1.48(\mathrm{~m}, 1 \mathrm{H}), 1.48-1.40(\mathrm{~m}, 1 \mathrm{H}), 1.40-1.27(\mathrm{~m}, 2 \mathrm{H})$, 1.31 (s, 9H); ${ }^{13} \mathrm{C}$ NMR (125 MHz, CD $\left.3 \mathrm{OD}\right): \delta 175.5$ (s), 165.5 (s, x2), 165.2 (s, x2), 159.3 (s), 132.8 (d, x2), 132.5 (d, x2), 129.0 (s, x2), 128.9 (s, x2), 128.8 (s, x2), 80.6 (s), 56.7 (d), 44.4 $(\mathrm{t}), 41.8(\mathrm{t}), 41.6(\mathrm{t}), 31.7(\mathrm{t}), 30.0(\mathrm{t}), 29.6(\mathrm{q}, \mathrm{x} 3), 25.7(\mathrm{t})$; Anal. Calc. for $\mathrm{C}_{27} \mathrm{H}_{31} \mathrm{~N}_{5} \mathrm{O}_{7} \bullet 3 \mathrm{H}_{2} \mathrm{O}$ (591.61): C 54.81, H 6.30, N 11.84; found: C 54.80, H 5.79, N 11.71; MS (ESI, $\left.\mathrm{CH}_{2} \mathrm{Cl}_{2} / \mathrm{MeOH} 9: 1\right): \mathrm{m} / \mathrm{z}(\%) 1098\left(20,[2 \mathrm{M}+\mathrm{Na}]^{+}\right), 1076\left(15,[2 \mathrm{M}+\mathrm{H}]^{+}\right), 576(8,[\mathrm{M}+$ $\left.\mathrm{K}]^{+}\right), 560\left(100,[\mathrm{M}+\mathrm{Na}]^{+}\right)$.

p-Octiphenyl 8. This compound was prepared from 9 in overall nine steps following previously reported procedures $(S 1)$.

$\mathbf{1}^{3}, \mathbf{2}^{\mathbf{3}}, \mathbf{3}^{\mathbf{2}}, \mathbf{4}^{\mathbf{3}}, \mathbf{5}^{\mathbf{2}}, \mathbf{6}^{\mathbf{3}}, \mathbf{7}^{\mathbf{2}}, \mathbf{8}^{\mathbf{3}}$-Octakis[NDI-Lys(Boc)- $\left.\mathrm{NH}_{2}\right]$-p-Octiphenyl 10. A solution of 8 (3.2 $\mathrm{mg}, 2.66 \mu \mathrm{mol})$, HATU (16.2 mg, $43.0 \mu \mathrm{mol})$, and 2,6-di-tert-butyl pyridine (29.0 $\mu 1,0.128$ $\mathrm{mmol})$ in DMF $(0.5 \mathrm{ml})$ was stirred for $30 \mathrm{~min}$ at $\mathrm{rt}$. Then, a solution of 7 (137.4 $\mathrm{mg}, 0.256$ $\mathrm{mmol})$ and 2,6-di-tert-butyl pyridine $(29.0 \mu \mathrm{l}, 0.128 \mathrm{mmol})$ in DMF $(2.0 \mathrm{ml})$ was added. After stirring for $24 \mathrm{~h}$ at $\mathrm{rt}$, the reaction mixture was concentrated in vacuo. Purification of the crude product by column chromatography $\left(\mathrm{CH}_{2} \mathrm{Cl}_{2} / \mathrm{MeOH} 5: 1\right)$ and PTLC $\left(\mathrm{CHCl}_{3} / \mathrm{MeOH}\right.$ $4: 1, R_{f}=0.5$ ) yielded HPLC-pure (YMC-Pack SIL 250 × $4.6 \mathrm{~mm}, \mathrm{CH}_{2} \mathrm{Cl}_{2} / \mathrm{MeOH}$ 9:1, $1 \mathrm{ml} / \mathrm{min}, R_{\mathrm{t}}=7.65 \mathrm{~min}$, Fig. S1A) $10(8.4 \mathrm{mg}, 59 \%)$ as a yellow solid. ${ }^{1} \mathrm{H}$ NMR $(300 \mathrm{MHz}$, $\left.\mathrm{CD}_{3} \mathrm{OD}\right): \delta 8.70-8.60(\mathrm{~m}, 4 \mathrm{H}), 8.58-8.32(\mathrm{~m}, 28 \mathrm{H}), 7.46-6.68$ (several $\left.\mathrm{m}, 26 \mathrm{H}\right), 5.65-5.45(\mathrm{~m}$, $8 \mathrm{H}), 4.50-4.05(\mathrm{~m}, 32 \mathrm{H}), 3.85-3.70(\mathrm{~m}, 4 \mathrm{H}), 3.70-3.45(\mathrm{~m}, 12 \mathrm{H}), 3.08-2.90(\mathrm{~m}, 16 \mathrm{H}), 2.35-$ $2.20(\mathrm{~m}, 16 \mathrm{H}), 1.60-1.40(\mathrm{~m}, 16 \mathrm{H}), 1.40-1.15$ (several $\mathrm{m}, 88 \mathrm{H})$.

$\mathbf{1}^{\mathbf{3}}, \mathbf{2}^{\mathbf{3}}, \mathbf{3}^{\mathbf{2}}, \mathbf{4}^{\mathbf{3}}, \mathbf{5}^{\mathbf{2}}, \mathbf{6}^{\mathbf{3}}, \mathbf{7}^{\mathbf{2}}, \mathbf{8}^{\mathbf{3}}$-Octakis(NDI-Lys-NH $\mathbf{H}_{\mathbf{2}}$ - $\boldsymbol{p}$-Octiphenyl 1. A solution of $\mathbf{1 0}$ (9.7 $\mathrm{mg}, 1.81$ $\mu \mathrm{mol})$ in TFA $/ \mathrm{CH}_{2} \mathrm{Cl}_{2} 1: 1(3 \mathrm{ml})$ was stirred for 90 min at rt, diluted with $\mathrm{CH}_{2} \mathrm{Cl}_{2}(5 \mathrm{ml})$, evaporated, dried in vacuo, then diluted with toluene and evaporated $(2 \mathrm{ml}, 3 \mathrm{x})$. Impurities were removed by solid-liquid extraction with $\mathrm{Et}_{2} \mathrm{O}(2 \mathrm{ml}, 4 \mathrm{x})$, hexane $(2 \mathrm{ml}, 4 \mathrm{x})$ and $\mathrm{CH}_{2} \mathrm{Cl}_{2}$ ( $2 \mathrm{ml}, 2 \mathrm{x}$ ) to give a yellow residual solid. The crude product was purified by RP-HPLC (YMC-Pack ODS-A, $250 \times 10 \mathrm{~mm}, \mathrm{MeOH} / \mathrm{H}_{2} \mathrm{O} / \mathrm{TFA}$ 80:19:1, $2 \mathrm{ml} / \mathrm{min}, R_{\mathrm{t}}=5.83 \mathrm{~min}$, Fig. S1B) to yield pure $1(6.0 \mathrm{mg}, 73 \%)$ as a yellow solid. UV-vis (TFE): 378 (126.8), 359 (117.0), 340 (53.0), 322 (86.9); UV-vis (10 mM HEPES, $100 \mathrm{mM} \mathrm{NaCl}, \mathrm{pH} 7.9$ ): 384 (56.8), 362 (70.0), 345 (72.3); CD (TFE): 386 (-8.0), 374 (+1.2), 367 (0.0), 355 (+7.8), 343 (+6.4); CD (10 mM HEPES, $100 \mathrm{mM} \mathrm{NaCl}, \mathrm{pH} 7.9): 391$ (-10.8), 351 (+13.4); ${ }^{1} \mathrm{H}$ NMR $(300 \mathrm{MHz}$, $\left.\mathrm{CD}_{3} \mathrm{OD}\right): \delta 8.68-8.60(\mathrm{~m}, 4 \mathrm{H}), 8.50-8.38(\mathrm{~m}, 28 \mathrm{H}), 7.46-6.68$ (several $\left.\mathrm{m}, 26 \mathrm{H}\right)$, 5.65-5.48 (m, $8 \mathrm{H}), 4.55-4.05(\mathrm{~m}, 32 \mathrm{H}), 3.85-3.70(\mathrm{~m}, 8 \mathrm{H}), 3.65-3.50(\mathrm{~m}, 24 \mathrm{H}), 3.00-2.85(\mathrm{~m}, 16 \mathrm{H}), 2.52-$ 
$2.30(\mathrm{~m}, 12 \mathrm{H}), 2.25-2.10(\mathrm{~m}, 4 \mathrm{H}), 1.75-1.60(\mathrm{~m}, 16 \mathrm{H}), 1.60-1.30$ (several $\mathrm{m}, 16 \mathrm{H})$; MS (ESI, $\left.\mathrm{CH}_{3} \mathrm{CN} / \mathrm{H}_{2} \mathrm{O} / \mathrm{MeOH} / \mathrm{AcOH} 37: 12: 5: 1\right): \mathrm{m} / \mathrm{z}(\%) 1520\left(10,[\mathrm{M}+3 \mathrm{H}]^{3+}\right), 1141(50,[\mathrm{M}+$ $\left.4 \mathrm{H}]^{4+}\right), 913\left(60,[\mathrm{M}+5 \mathrm{H}]^{5+}\right), 761\left(100,[\mathrm{M}+6 \mathrm{H}]^{6+}\right), 652\left(40,[\mathrm{M}+7 \mathrm{H}]^{7+}\right)($ Fig. S2).
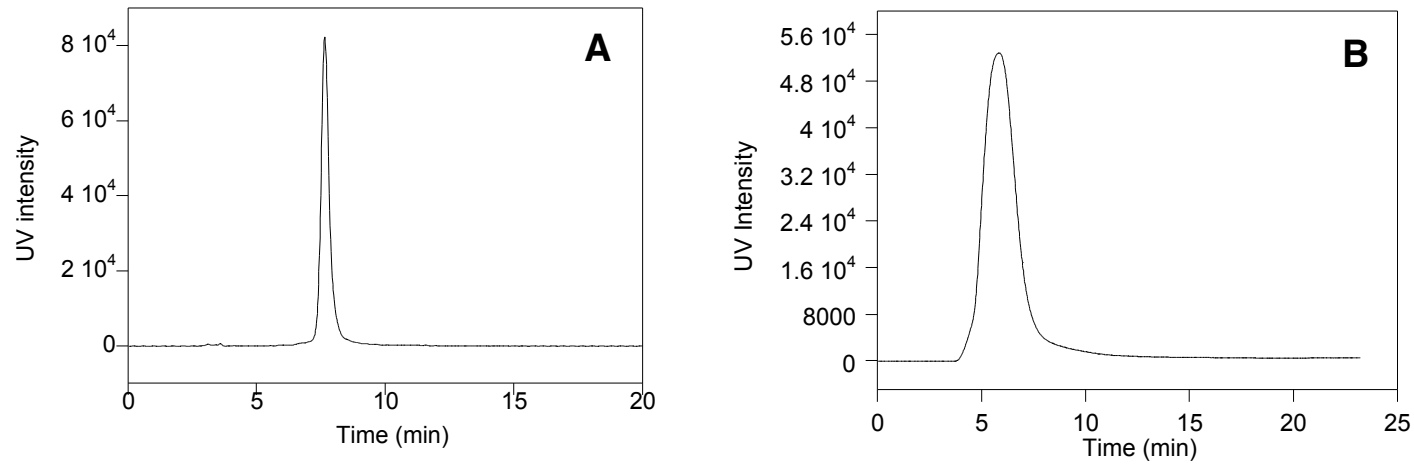

Figure S1. HPLC of (A) protected (10) and (B) deprotected target molecule $\mathbf{1}$ (detection at $254 \mathrm{~nm})$.

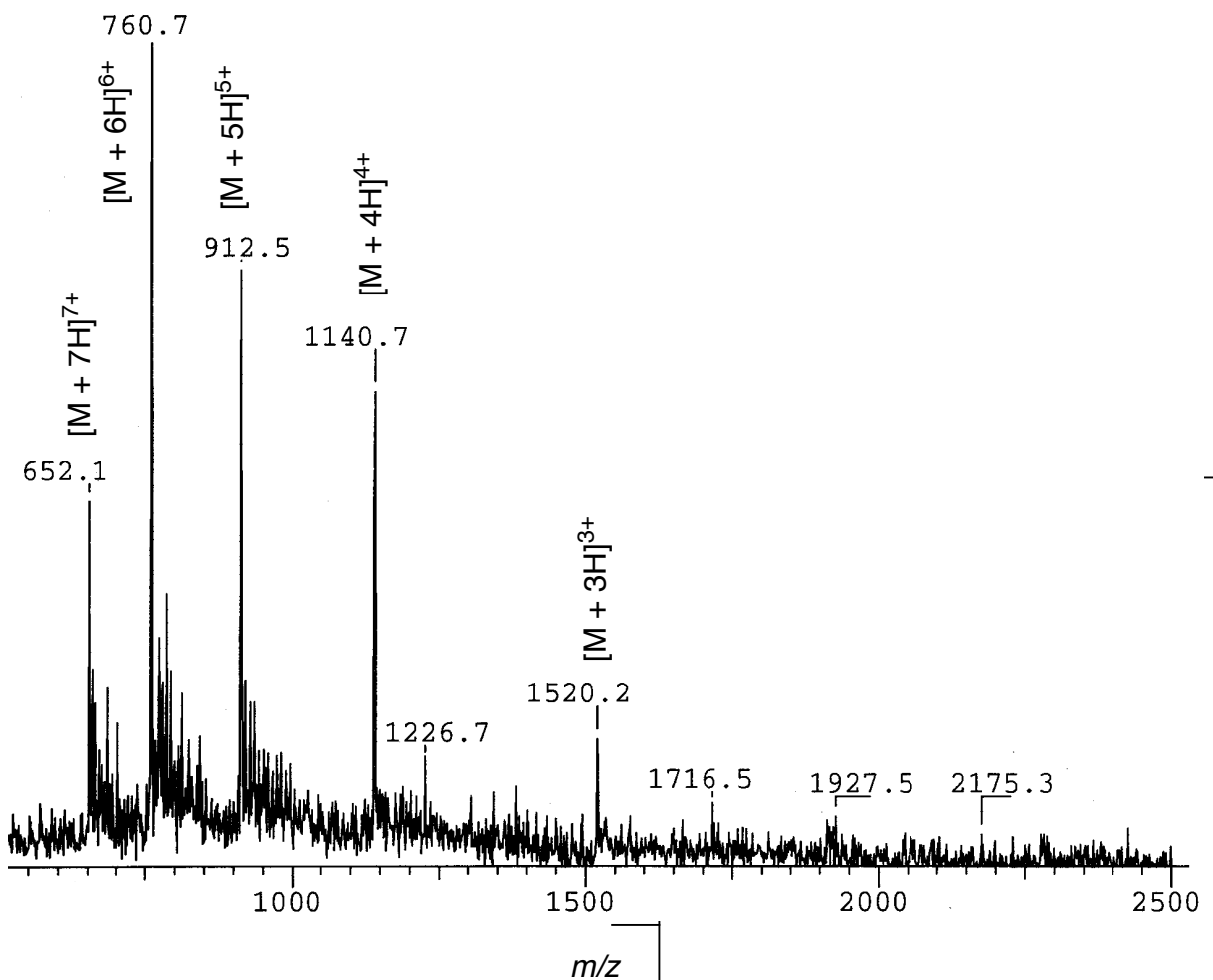

Figure S2. ESI-MS of target molecule 1 under denaturating conditions. 
Synthesis of Ligands.
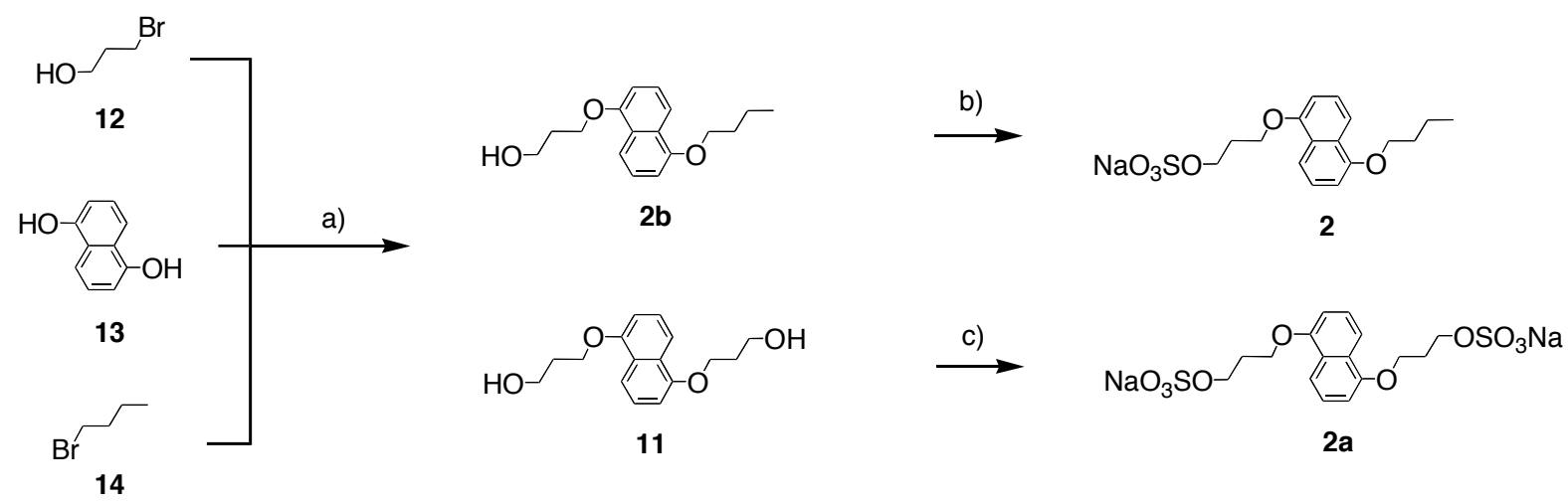

Scheme S2. a) $\mathrm{Cs}_{2} \mathrm{CO}_{3}$, acetone, $24 \mathrm{~h}, \mathrm{rt}, 36 \% \mathbf{2 b}, 36 \%$ 11; b) chlorosulfonic acid, $\mathrm{CH}_{2} \mathrm{Cl}_{2}, 75$ min, $0{ }^{\circ} \mathrm{C}, 73 \%$; c) chlorosulfonic acid, $\mathrm{CH}_{2} \mathrm{Cl}_{2}, 75 \mathrm{~min}, 0{ }^{\circ} \mathrm{C}, 82 \%$.

Bu-DAN-OH 2b and HO-DAN-OH 11. To $\mathrm{Cs}_{2} \mathrm{CO}_{3}(4.068 \mathrm{~g}, 12.49 \mathrm{mmol})$ in acetone (25 $\mathrm{ml}), 12(0.34 \mathrm{ml}, 3.75 \mathrm{mmol}), \mathbf{1 3}(500 \mathrm{mg}, 3.12 \mathrm{mmol})$ and $14(0.40 \mathrm{ml}, 3.75 \mathrm{mmol})$ were added. After stirring at $\mathrm{rt}$ for $24 \mathrm{~h}$ under Ar, the reaction mixture was concentrated in vacuo, dissolved in $\mathrm{CH}_{2} \mathrm{Cl}_{2}(200 \mathrm{ml})$, washed with water $(20 \mathrm{ml}), 1 \mathrm{M} \mathrm{HCl}(20 \mathrm{ml})$, water $(20 \mathrm{ml})$ and brine $(20 \mathrm{ml})$, dried over $\mathrm{Na}_{2} \mathrm{SO}_{4}$ and evaporated in vacuo. The crude product was purified by column chromatography $\left(\mathrm{CH}_{2} \mathrm{Cl}_{2} / \mathrm{MeOH}\right.$, gradient 40:1-20:1-10:1) to give pure $\mathbf{2 b}$ (307 mg, 36\%) and $\mathbf{1 1}(310 \mathrm{mg}, 36 \%)$ as colorless solids. 2b: m.p.: 93.0-93.5 ${ }^{\circ} \mathrm{C}$; IR: v 3244 (m), 3024 (w), 2931 (m), 2870 (m), 1592 (s), 1511 (s), 1416 (s), 1385 (s), 1269 (s), 1067 (s), $775(\mathrm{~s}) ;{ }^{1} \mathrm{H}$ NMR $\left(300 \mathrm{MHz}, \mathrm{CD}_{3} \mathrm{OD}\right): \delta 7.82\left(\mathrm{~d},{ }^{3} J(\mathrm{H}, \mathrm{H})=8.5 \mathrm{~Hz}, 1 \mathrm{H}\right), 7.77\left(\mathrm{~d},{ }^{3} J\right.$ $(\mathrm{H}, \mathrm{H})=8.5 \mathrm{~Hz}, 1 \mathrm{H}), 7.35-7.27(\mathrm{~m}, 2 \mathrm{H}), 6.85\left(\mathrm{~d},{ }^{3} J(\mathrm{H}, \mathrm{H})=8.5 \mathrm{~Hz}, 1 \mathrm{H}\right), 6.82\left(\mathrm{~d},{ }^{3} J(\mathrm{H}, \mathrm{H})=\right.$ $8.4 \mathrm{~Hz}, 1 \mathrm{H}), 4.23\left(\mathrm{t},{ }^{3} J(\mathrm{H}, \mathrm{H})=6.0 \mathrm{~Hz}, 2 \mathrm{H}\right), 4.11\left(\mathrm{t},{ }^{3} J(\mathrm{H}, \mathrm{H})=6.4 \mathrm{~Hz}, 2 \mathrm{H}\right), 3.86\left(\mathrm{t},{ }^{3} J(\mathrm{H}, \mathrm{H})\right.$ $=6.4 \mathrm{~Hz}, 2 \mathrm{H}), 2.18-2.10(\mathrm{~m}, 2 \mathrm{H}), 1.93-1.83(\mathrm{~m}, 2 \mathrm{H}), 1.64-1.54(\mathrm{~m}, 2 \mathrm{H}), 1.00\left(\mathrm{t},{ }^{3} J(\mathrm{H}, \mathrm{H})=\right.$ $7.3 \mathrm{~Hz}) ;{ }^{13} \mathrm{C}$ NMR $\left(100 \mathrm{MHz}, \mathrm{CD}_{3} \mathrm{OD}\right): \delta 156.5(\mathrm{~s}), 156.3(\mathrm{~s}), 128.6(\mathrm{~s}, \mathrm{x} 2), 126.8(\mathrm{~s}), 126.7$ $(\mathrm{s}), 115.8(\mathrm{~s}), 115.6(\mathrm{~s}), 107.0(\mathrm{~d}, \mathrm{x} 2), 69.5(\mathrm{t}), 66.5(\mathrm{t}), 60.5(\mathrm{t}), 34.0(\mathrm{t}), 33.2(\mathrm{t}), 21.2(\mathrm{t}), 15.0$ (q); MS (EI): m/z (\%) $274\left(68, \mathrm{M}^{+*}\right), 218$ (31), 160 (100), 131 (27). 11: m.p.: 141.5-142.0 ${ }^{\circ} \mathrm{C}$; UV-vis (DMSO): 294 (11.7), 314 (10.2), 327 (7.8); IR: v 3357 (m), 3305 (m), 2948 (m), 2874 (m), 1591 (s), 1513 (s), 1415 (s), 1387 (s), 1266 (s), 1063 (s), 1054 (s), 772 (s); ${ }^{1} \mathrm{H}$ NMR $\left(400 \mathrm{MHz}, \mathrm{CD}_{3} \mathrm{OD}\right): \delta 7.80\left(\mathrm{~d},{ }^{3} J(\mathrm{H}, \mathrm{H})=8.6 \mathrm{~Hz}, 2 \mathrm{H}\right), 7.34\left(\mathrm{dd},{ }^{3} J(\mathrm{H}, \mathrm{H})=8.6 \mathrm{~Hz},{ }^{3} J(\mathrm{H}, \mathrm{H})\right.$ $=7.6 \mathrm{~Hz}, 2 \mathrm{H}), 6.88\left(\mathrm{~d},{ }^{3} J(\mathrm{H}, \mathrm{H})=7.6 \mathrm{~Hz}, 2 \mathrm{H}\right), 4.25\left(\mathrm{t},{ }^{3} J(\mathrm{H}, \mathrm{H})=6.0 \mathrm{~Hz}, 2 \mathrm{H}\right), 3.88\left(\mathrm{t},{ }^{3} J\right.$ $(\mathrm{H}, \mathrm{H})=6.0 \mathrm{~Hz}, 2 \mathrm{H}), 2.15$ (quint, $\left.{ }^{3} J(\mathrm{H}, \mathrm{H})=6.0 \mathrm{~Hz}, 2 \mathrm{H}\right) ;{ }^{13} \mathrm{C} \mathrm{NMR}\left(100 \mathrm{MHz}, \mathrm{CD}_{3} \mathrm{OD}\right): \delta$ 155.8 (s, x2), 128.1 (s, x2), 126.4 (d, x2), 115.4 (d, x2), 106.7 (d, x2), 66.3 (t, x2), 60.3 (t, x2), 33.5 (t, x2); MS (EI): $276\left(53, \mathrm{M}^{+\bullet}\right), 160$ (100), 131 (29).

Bu-DAN-OSO $\mathrm{H}_{3}$ 2. To a solution of chlorosulfonic acid $(14 \mu 1,0.21 \mathrm{mmol})$ in anhydrous $\mathrm{CH}_{2} \mathrm{Cl}_{2}(2 \mathrm{ml})$ at $0{ }^{\circ} \mathrm{C}, \mathbf{2} \mathbf{b}(48.1 \mathrm{mg}, 0.17 \mathrm{mmol})$ in anhydrous $\mathrm{CH}_{2} \mathrm{Cl}_{2}(5 \mathrm{ml})$ was added over 15 minutes. The mixture was stirred and purged with nitrogen for $1 \mathrm{~h}$ at $0{ }^{\circ} \mathrm{C}$, then warmed to $\mathrm{rt}$ and evaporated in vacuo. The crude product was washed with hexane $(10 \mathrm{ml}, 2 \mathrm{x})$ and purified by reverse-phase column chromatography $\left(\mathrm{H}_{2} \mathrm{O}, R_{\mathrm{f}}=0.3\right)$ to give pure $2(44.8 \mathrm{mg}$, 73\%) as a grey powder. m.p.: 204.0-204.5 ${ }^{\circ} \mathrm{C}$; IR: $v 3384(\mathrm{~m}), 2955(\mathrm{~m}), 1658(\mathrm{~s}), 1594(\mathrm{~s})$, 1511 (s), 1417 (s), 1248 (s), 1196 (s), 1050 (s), 760 (s); ${ }^{1} \mathrm{H}$ NMR (300 MHz, CD ${ }_{3} \mathrm{OD}$ ): $\delta 7.82$ 
$\left(\mathrm{d},{ }^{3} J(\mathrm{H}, \mathrm{H})=8.3 \mathrm{~Hz}\right), 7.80\left(\mathrm{~d},{ }^{3} J(\mathrm{H}, \mathrm{H})=7.9 \mathrm{~Hz}, 1 \mathrm{H}\right), 7.40-7.30(\mathrm{~m} \mathrm{2H}), 6.88\left(\mathrm{~d},{ }^{3} J(\mathrm{H}, \mathrm{H})=\right.$ $6.8 \mathrm{~Hz}, 1 \mathrm{H}), 6.86\left(\mathrm{~d},{ }^{3} J(\mathrm{H}, \mathrm{H})=7.0 \mathrm{~Hz}, 1 \mathrm{H}\right), 4.34\left(\mathrm{t},{ }^{3} J(\mathrm{H}, \mathrm{H})=6.2 \mathrm{~Hz}, 2 \mathrm{H}\right), 4.26\left(\mathrm{t},{ }^{3} J(\mathrm{H}, \mathrm{H})\right.$ $=6.0 \mathrm{~Hz}, 2 \mathrm{H}), 4.13\left(\mathrm{t},{ }^{3} J(\mathrm{H}, \mathrm{H})=6.4 \mathrm{~Hz}, 2 \mathrm{H}\right) 2.31$ (quint, $\left.{ }^{3} \mathrm{~J}(\mathrm{H}, \mathrm{H})=6.2 \mathrm{~Hz}, 2 \mathrm{H}\right), 1.96-1.86$ $(\mathrm{m}, 2 \mathrm{H}), 1.67-1.55(\mathrm{~m}, 2 \mathrm{H}), 1.04\left(\mathrm{t},{ }^{3} J(\mathrm{H}, \mathrm{H})=5.7 \mathrm{~Hz}, 3 \mathrm{H}\right) ;{ }^{13} \mathrm{C} \mathrm{NMR}\left(75 \mathrm{MHz}, \mathrm{CD}_{3} \mathrm{OD}\right): \delta$ $156.2(\mathrm{~s}), 155.9$ (s), 128.4 (s, x2), 126.6 (s), 126.5 (s), 115.8 (s), 115.4 (s), 107.0 (d), 106.9 $(\mathrm{d}), 69.4(\mathrm{t}), 66.9(\mathrm{t}), 66.0(\mathrm{t}), 32.9(\mathrm{t}), 30.9(\mathrm{t}), 21.0(\mathrm{t}), 15.0(\mathrm{q}) ; \mathrm{MS}\left(\mathrm{ESI}, \mathrm{CH}_{2} \mathrm{Cl}_{2} / \mathrm{MeOH}\right.$ 50:1): $\mathrm{m} / \mathrm{z}(\%) 353\left(100,[\mathrm{M}-\mathrm{H}]^{-}\right)$.

$\mathrm{NaO}_{3} \mathrm{SO}-\mathrm{DAN}-\mathrm{OSO}_{3} \mathrm{Na} 2 \mathrm{2a}$. To a solution of chlorosulfonic acid $(10.6 \mu 1,0.16 \mathrm{mmol})$ in anhydrous THF $(2 \mathrm{ml})$ at $0{ }^{\circ} \mathrm{C}, \mathbf{1 1}(20 \mathrm{mg}, 72.4 \mu \mathrm{mol})$ in anhydrous THF $(5 \mathrm{ml})$ was added over 12 minutes. The mixture was stirred and purged with nitrogen for $1 \mathrm{~h}$ at $0{ }^{\circ} \mathrm{C}$, then warmed to rt, diluted with water $(2 \mathrm{ml})$, neutralized with $1 \mathrm{M} \mathrm{NaOH}$ and evaporated in vacuo. Hexane soluble impurities were removed by solid-liquid extraction $(10 \mathrm{ml}, 2 \mathrm{x})$ and the residue was purified by reverse-phase column chromatography $\left(\mathrm{H}_{2} \mathrm{O} / \mathrm{MeOH} 1: 1, R_{\mathrm{f}}=0.6\right)$ to give pure 2a $(28.5 \mathrm{mg}, 82 \%)$ as a colorless powder. m.p.: $>200{ }^{\circ} \mathrm{C}$; IR: $v 3457(\mathrm{~m}), 2870$ (w), $2943(\mathrm{~m}), 1594(\mathrm{~m}), 1511(\mathrm{~m}), 1418$ (s), 1210 (s), 1063 (s), 959 (s), 835 (s), 769 (s), 617 (s); ${ }^{1} \mathrm{H}$ NMR $\left(400 \mathrm{MHz}, \mathrm{D}_{2} \mathrm{O}\right): \delta 7.71\left(\mathrm{~d},{ }^{3} J(\mathrm{H}, \mathrm{H})=8.3 \mathrm{~Hz}, 2 \mathrm{H}\right), 7.31\left(\mathrm{dd},{ }^{3} J(\mathrm{H}, \mathrm{H})=8.3 \mathrm{~Hz}\right.$, $\left.{ }^{3} J(\mathrm{H}, \mathrm{H})=7.6 \mathrm{~Hz}, 2 \mathrm{H}\right), 6.90\left(\mathrm{~d},{ }^{3} J(\mathrm{H}, \mathrm{H})=7.6 \mathrm{~Hz}, 2 \mathrm{H}\right), 4.19\left(\mathrm{t},{ }^{3} J(\mathrm{H}, \mathrm{H})=6.0 \mathrm{~Hz}, 2 \mathrm{H}\right), 4.16$ $\left(\mathrm{t},{ }^{3} J(\mathrm{H}, \mathrm{H})=6.0 \mathrm{~Hz}, 2 \mathrm{H}\right), 2.13$ (quint, $\left.{ }^{3} \mathrm{~J}(\mathrm{H}, \mathrm{H})=6.0 \mathrm{~Hz}, 2 \mathrm{H}\right) ;{ }^{13} \mathrm{C}$ NMR $\left(100 \mathrm{MHz}, \mathrm{D}_{2} \mathrm{O}\right): \delta$ $153.7(\mathrm{~s}, \mathrm{x} 2), 126.3(\mathrm{~s}, \mathrm{x} 2), 125.9(\mathrm{~d}, \mathrm{x} 2), 114.2(\mathrm{~d}, \mathrm{x} 2), 107.0(\mathrm{~d}, \mathrm{x} 2), 66.2(\mathrm{t}, \mathrm{x} 2), 65.1(\mathrm{t}, \mathrm{x} 2)$, $28.5(\mathrm{t}, \mathrm{x} 2) ; \mathrm{MS}(\mathrm{ESI}, \mathrm{MeOH}): \mathrm{m} / \mathrm{z}(\%) 458\left(100,[\mathrm{M}-\mathrm{Na}]^{-}\right), 218\left(38,[\mathrm{M}-2 \mathrm{Na}]^{2-}\right)$.

\section{Ion Transport Assays.}

Stock Solutions. Stock solutions were prepared at $c_{\text {STOCK }}=100 \times c$ of interest in hydrophilic organic solvents or buffer (2b: DMSO. 2a: DMSO/ $\mathrm{H}_{2} \mathrm{O}$ 1:1. 2: $\mathrm{DMSO} / \mathrm{H}_{2} \mathrm{O}$ 1:1. 1: DMSO or DMSO/THF 1:1 (use of MeOH or water as solvents resulted in reduced activity)). All concentrations were confirmed by UV-vis spectroscopy, $\mathrm{pH}$ was adjusted spectroscopically following a previously described method $(S 3)$.

EYPC-LUVsつHPTS. A solution of EYPC (25 mg) in $\mathrm{CHCl}_{3} / \mathrm{MeOH} 1: 1$ was dried using a rotory evaporator and then under vacuum $(>2 \mathrm{~h})$ to form a thin film. Hydration for $>30 \mathrm{~min}(1$ mM HPTS, $10 \mathrm{mM}$ HEPES, $100 \mathrm{mM} \mathrm{NaCl}, \mathrm{pH} 7.0)$, freeze-thaw cycles $(\geq 5 \mathrm{x})$, extrusion $(\geq$ $15 \mathrm{x}, 100 \mathrm{~nm}$ polycarbonate membranes), external buffer exchange (Sephadex G-50 column, $10 \mathrm{mM}$ HEPES, $100 \mathrm{mM} \mathrm{NaCl}, \mathrm{pH}$ 7.0) and dilution to $6 \mathrm{ml}$ gave EYPC-LUVsつHPTS: $\sim 2.5$ mM EYPC, inside: $1 \mathrm{mM}$ HPTS, $10 \mathrm{mM}$ HEPES, $100 \mathrm{mM} \mathrm{NaCl}, \mathrm{pH} 7.0$, outside: $10 \mathrm{mM}$ HEPES, $100 \mathrm{mM} \mathrm{NaCl}, \mathrm{pH} 7.0$ (adapted from (S2) and (S4)).

Ligand Gating in EYPC-LUVsつHPTS. EYPC-LUVsつHPTS ( $25 \mu 1)$ were added to gently stirred, thermostated buffer (1975 $\mu 1,10 \mathrm{mM}$ HEPES, $100 \mathrm{mM} \mathrm{NaCl}$ (or other salts, see Figs. $\mathrm{S} 3 \mathrm{C}$ and $\mathrm{S} 3 \mathrm{D}), \mathrm{pH}=7.0)$ in a fluorescence cuvette $(\mathrm{t}=0 \mathrm{sec})$. The time course of HPTS fluorescence emission intensity, $F_{\mathrm{t}}$, was followed at $\lambda_{\mathrm{em}}=510 \mathrm{~nm}\left(\lambda_{\mathrm{ex}}{ }^{1}=450 \mathrm{~nm}, \mathrm{pH}\right.$-control: $\left.\lambda_{\mathrm{ex}}{ }^{2}=405 \mathrm{~nm}\right)$ during the addition of $0.5 \mathrm{M} \mathrm{NaOH}(20 \mu 1)$, ligands $(20 \mu 1 \mathbf{2}, \mathbf{2} \mathbf{a}$ or $\mathbf{2 b}$ in DMSO (or nucleotides, Fig. S3B) at varied concentration), pore (20 $\mu 1$ 1) in DMSO at varied concentration) and gramicidin A (20 $\mu \mathrm{l}$ of $20 \mu \mathrm{M}$ in water) at the end of each experiment. Fluorescence time courses were normalized to fractional emission intensity $I^{\mathrm{m}}$ using equation [S1] 


$$
I^{\mathrm{n}}=\left(F_{\mathrm{t}}-F_{0}\right) /\left(F_{\infty}-F_{0}\right)
$$

where $F_{0}=F_{\mathrm{t}}$ at pore addition, $F_{\infty}=F_{\mathrm{t}}$ at saturation after complete leakage. The obtained $I^{\mathrm{n}}(\mathrm{t})$ was calibrated to $I_{\mathrm{MAX}}^{\mathrm{m}}$ using

$$
I(\mathrm{t})=I^{\mathrm{n}}(\mathrm{t}) / I_{\text {MAX }}^{\mathrm{n}}
$$

where $I_{\text {MAX }}^{\mathrm{n}}$ is the reference value of interest, e.g., the highest activity in a given series before addition of gramicidin A (Fig. S3 and 2A). Fractional pore activity $I$ was determined using equation $[\mathrm{S} 3]$

$$
I=I^{\mathrm{n}} / I_{\mathrm{MAX}}^{\mathrm{m}}
$$

where $I_{\text {MAX }}^{\mathrm{n}}$ is the reference value at a given time and $I^{\mathrm{n}}$ the value obtained at the same time for a given experimental variation. Fractional activities of pore $I$ at $100 \mathrm{sec}$ were determined as a function of ligand concentration and fitted to the Hill equation [S4] to give effective concentrations $E C_{50}$ and the Hill coefficient $n$.

$$
I=I_{\infty}+\left(I_{0}-I_{\infty}\right) /\left\{1+\left([\text { ligand }] / E C_{50}\right)^{n}\right\}
$$

where $I_{0}$ is $I$ without ligand, and $I_{\infty}$ is $I$ with excess ligand (Fig. 2B) (adapted from, e.g., (S5)).
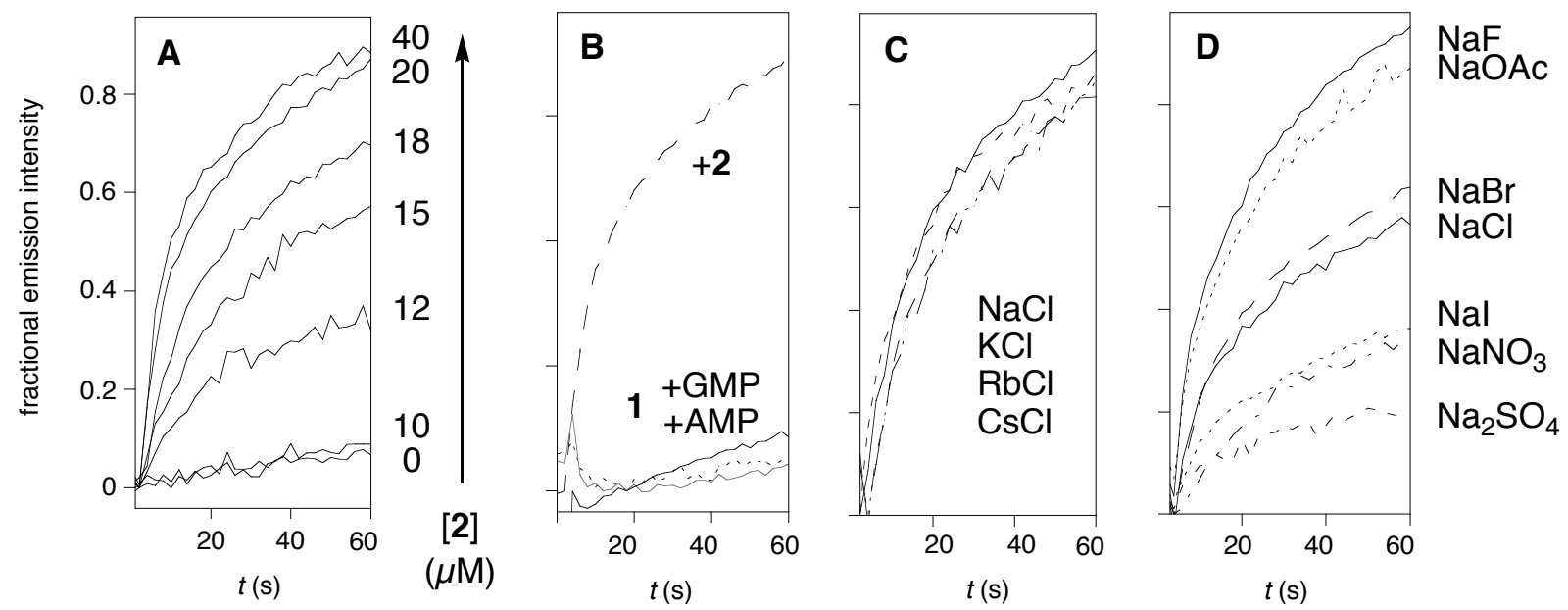

Figure S3. Dependence of the activity of NDI pore $1(1.6 \mu \mathrm{M})$ in EYPC-LUVsつHPTS on concentration (A, 2) and nature of ligands (B, 5.0 mM GMP, $2.5 \mathrm{mM}$ AMP (grey), compared to $20 \mu \mathrm{M} 2$ (dashed) and ligand-free 1 (dotted)) and dependence of DAN-NDI complexes $\mathbf{1}^{n} \cdot \mathbf{2}^{m}(1.6 \mu \mathrm{M} \mathrm{1,20 \mu} \mu \mathrm{M} \mathrm{2})$ on nature and size of extravesicular cations (C) and anions (D, $I_{\text {MAX }}$ for NaF). Fluorescence time courses obtained following the procedure described above until normalization with eq [S1] and [S2].

Fig. S3, comments: S3B: For representative NDI-nucleotide complexes, please see (S6). S3C: Independence on external cations suggested that channels are anion selective. S3D: Dependence on external anions suggested that channels are anion selective and gave selectivity sequence. For comparison of ion selectivities in BLMs and LUVs, please see (S7). 
EYPC-LUVs $\supset$ ANTS/DPX. This assay was applied as described in (S8), internal buffer: $12.5 \mathrm{mM}$ ANTS, $45.0 \mathrm{mM}$ DPX, $5 \mathrm{mM}$ TES, $20 \mathrm{mM} \mathrm{KCl}, \mathrm{pH}=7.0$; external buffer: $5 \mathrm{mM}$ TES, $100 \mathrm{mM} \mathrm{KCl}, \mathrm{pH}=7.0$ or 7.9. No ANTS/DPX efflux was observed with $\mathbf{1}^{n} \cdot \mathbf{2}^{m}(0-1.6$ $\mu \mathrm{M} 1,0-50 \mu \mathrm{M} 2$ ).

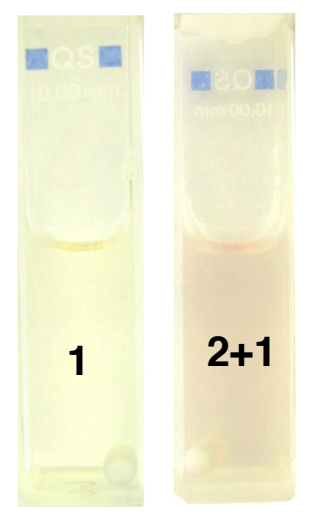

Figure S4. Example for naked-eye detection of CT complexes $\mathbf{1}^{n} \cdot \mathbf{2}^{\boldsymbol{m}}$.

(S1) Baumeister, B.; Sakai, N.; Matile, S. Org. Lett. 2001, 3, 4229-4232.

(S2) Weiss, L.A.; Sakai, N.; Ghebremariam, B.; Ni, C.; Matile, S. J. Am. Chem. Soc. 1997, 119, 12142-12149.

(S3) Das, G.; Onouchi, H.; Yashima, E.; Sakai, N.; Matile, S. ChemBioChem 2002, 3, 1089-1096.

(S4) Sakai, N.; Matile, S. J. Am. Chem. Soc. 2003, 125, 14348-14356.

(S5) Gorteau, V.; Perret, F.; Bollot, G.; Mareda, J.; Lazar, A. N.; Coleman, A. W.; Tran, D.-H.; Sakai, N.; Matile, S. J. Am. Chem. Soc. 2004, 126, 13592-13593.

(S6) Lee, J.; Guelev, V.; Sorey, S.; Hoffman, D. W.; Iverson, B. L. J. Am. Chem. Soc. 2004, 126, 14036-14042.

(S7) Sakai, N.; Houdebert, D.; Matile, S. Chem. Eur. J. 2003, 9, 223-232.

(S8) Talukdar, P.; Sakai, N.; Sordé, N.; Gerard, D.; Cardona, V. M. F.; Matile, S. Bioorg. Med. Chem. 2004, 12, 1325-1336. 